\title{
Reflexiones en torno a la forma urbana en la experiencia francesa de renovación urbana de los polígonos de vivienda masiva
}

\author{
Sergio García-Pérez ${ }^{1}$ \\ Recibido: 15-11-2020 | en su versión final: 03-02-2021
}

Resumen

\begin{abstract}
Hasta el momento, las estrategias de renovación, regeneración o rehabilitación de polígonos de vivienda masiva efectuadas en España tienden a no cuestionar su forma urbana. Sin embargo, el carácter planificado de los polígonos y la mayor cantidad de espacio libre suponen una gran oportunidad para la promoción de una mayor calidad urbana. El carácter sectorial de la mayoría de operaciones realizadas, al obviar esta dimensión, no ha permitido optimizar el potencial morfológico en la regeneración urbana de los polígonos de vivienda. En contraste, existen experiencias europeas más avanzadas cuyo estudio podría ayudar a mejorar las estrategias de regeneración en nuestro país. Entre todas ellas, la renovación de polígonos franceses ha sido un laboratorio de experimentación para el estudio de las transformaciones morfológicas. Más allá de las particularidades del contexto y de las limitaciones de la experiencia francesa, el objetivo de este artículo es contrastar en qué medida la transformación de los espacios libres de algunos ejemplos de regeneración de polígonos franceses ha conseguido promover una mayor calidad urbana. Para ello, la investigación aplica la metodología de diagnóstico UR-Hesp sobre algunos casos de la región de île-de-France. Su aplicación permite reflexionar en torno a las ventajas de la aproximación mixta urbano-edificatoria francesa, sin obviar las advertencias que desde ámbitos como la sociología están comenzando a surgir al evaluar las primeras experiencias. Aprender de los aciertos y errores de una experiencia internacional podrá tener grandes beneficios en las futuras estrategias de regeneración urbana integrada en nuestro país.
\end{abstract}

Palabras clave: Calidad urbana; morfología urbana; espacio libre; reparcelación

Citación

García-Pérez, S. (2021). Reflexiones en torno a la forma urbana en la experiencia francesa de renovación urbana de los polígonos de vivienda masiva, ACE: Architecture, City and Environment, 16(46), 9876. DOI: http://dx.doi.org/10.5821/ace.16.46.9876

\section{Discussions on Urban Form in the French Experience of urban Renewal of Mass Housing Estates}

Abstract

\begin{abstract}
To date, the strategies for renewal, regeneration, or retrofitting of mass housing estates carried out in Spain tend not to question their urban form. However, the planned nature of the housing estates and the greater amount of open space represent a great opportunity for the promotion of greater urban quality. The sectoral nature of most of the actions carried out, by ignoring this dimension, has not allowed to optimise the morphological potential in the urban regeneration of housing estates. In contrast, there are more advanced European experiences whose study could help to improve regeneration strategies in our country. Among all of them, the renewal of French housing estates has been an important laboratory for the study of morphological transformations. Beyond the particularities of the context and the limitations of the French experience, the aim of this paper is to contrast the extent to which the transformation of the open spaces of some examples of regeneration of French housing estates has succeeded in promoting greater urban quality. To this end, the research applies the UR-Hesp diagnosis methodology to a number of cases in the île-de-France region. Its application allows reflecting on the advantages of the mixed urban-building French approach, without ignoring the recommendations that are beginning to emerge from fields such as sociology when evaluating the first experiences. Learning from the successes and failures of an international experience could have great benefits for future integrated urban regeneration strategies in our country.
\end{abstract}

Keywords: Urban quality; urban morphology; open space; replot

Dr. Arquitecto. Profesor asociado, Departamento de Arquitectura, Universidad de Zaragoza (ORCID ID: 0000-0002-1672-4965, Scopus Author ID: 57192872944, Researcher ID: I-4368-2016). Correo de contacto: sgarciap@unizar.es

ACE, 16 (4.6) CC BY-ND 3.0 ES | UPC Barcelona, España | Reflexiones en torno a la forma urbana en la experiencia francesa de renovación urbana de los polígonos de vivienda masiva. DOI: http://dx.doi.org/10.5821/ace.16.46.9876 


\section{Introducción}

Desde su creación, los polígonos de vivienda han sido objeto de un intenso debate urbanístico. Entre la literatura encontramos visiones en ocasiones divergentes que han focalizado sobre su emergencia y su significado histórico, o bien sobre su acelerada obsolescencia y los problemas físicos, sociales y económicos que rápidamente desarrollaron (Díez Medina y Monclús, 2020). En la actualidad, el debate en torno a los polígonos sigue vigente, centrado sobre todo en sus necesidades de actualización. Más aún en España, donde el predominante régimen de tenencia en propiedad de los polígonos ha propiciado que durante los últimos años haya comenzado a producirse un proceso de renovación de la población que puede desencadenar cambios radicales en el presente y en el futuro de los conjuntos (Díez de Pablo, 2015).

Sin embargo, el término 'polígonos de vivienda masiva' admite diversos significados en diferentes contextos y situaciones. A pesar de las diferencias entre países, el término está relacionado con los 'mass housing estates' de influencia anglosajona o de 'grands ensembles' según la literatura francófona (Monclús y Díez Medina, 2016). Esta investigación adopta un significado amplio del término 'polígonos de vivienda', el cual expresa un nuevo paradigma del desarrollo de la ciudad en una época determinada: características comunes de planificación, urbanidad, apariencia, tipologías, tenencia, función y localización (Wassenberg, 2012). Y es que, a pesar de las especificidades de cada contexto "nunca ha habido un periodo en la construcción de vivienda en el que la semejanza entre países haya sido tan grande" (Wassenberg, 2013, p. 169), en el que los polígonos constituyen un paisaje urbano residencial amplio, heredero del Movimiento Moderno (Díez Medina, 2015).

A pesar de la indiscutible homogeneidad de esta forma urbana tan característica, cincuenta años después de su construcción, los polígonos han experimentado diversas trayectorias de evolución. Factores socioeconómicos, como el tipo de población que ha ocupado los polígonos a lo largo de su vida útil, u otros físicos, como los diversos procesos de transformación internos o externos experimentados, han contribuido a su diversificación. Frente a la crítica generalista que en ocasiones ha acompañado el debate en torno a los polígonos de vivienda, recientes investigaciones han incidido en la necesidad de realizar aproximaciones específicas (Díez Medina y Monclús, 2017; Rowlands et al., 2009).

Por ello, ante los procesos de obsolescencia que experimentan muchos de los polígonos, uno de los retos actuales a los que se enfrenta este legado de la modernidad es la búsqueda de actualizaciones integradas, basado en el reconocimiento de problemas y oportunidades específicos en cada caso concreto (Díaz Gómez et al., 2019). Sin embargo, en la mayoría de los casos, las intervenciones en España han sido sectoriales al resolver de forma genérica muchos de los problemas técnicos que los edificios han presentado - constructivos, medioambientales y funcionales_- (Cervero Sánchez, 2020; Cocco y López, 2015; Daumal et al., 2018). De hecho, tal como reconoce el trabajo de A. Martínez Gutiérrez (2017) la experiencia de regeneración en España ha tendido a no cuestionar la forma urbana de los polígonos de vivienda. Frente a esta situación, ha surgido una línea crítica que trata de poner en valor las aproximaciones específicas urbanas (García-Pérez, 2019; Higueras García, 2015; Sendra, 2016). A pesar de las singularidades de cada una de ellas, todas coinciden en detectar no solo la oportunidad que supone intervenir sobre los espacios libres de los polígonos, sino también la relevancia de este tipo de actuaciones. Por un lado, porque los espacios libres fueron una de las aportaciones más importantes a la cultura arquitectónica contemporánea, como alternativa al modelo de calles y plazas de la ciudad tradicional (Castrillo Romón et al., 2016). Por otro, por la ineludible necesidad de cuestionar la visión optimista que el urbanismo funcionalista depositó sobre los espacios libres, fundada sobre los principios higienistas e igualitarios (Van Es et al., 2015), que pronto se vio superada por la crítica urbanística (Díez Medina y Monclús, 2020). Y es que la regeneración de esta forma urbana no puede obviar la necesidad y oportunidad de reconfiguración

ACE, 16 (4.6) CC BY-ND 3.0 ES | UPC Barcelona, España | Reflexiones en torno a la forma urbana en la experiencia francesa de renovación urbana de los polígonos de vivienda masiva. DOI: http://dx.doi.org/10.5821/ace.16.46.9876 
de los espacios libres. Tanto su carácter planificado como la disponibilidad de espacio, en muchas ocasiones residual, facilita las aproximaciones en esta línea de trabajo.

A pesar de que la gran mayoría de intervenciones en España no intervienen desde esta óptica, sí existen algunos ejemplos que han transformado la forma urbana de los espacios libres. Es el caso del conjunto de San Martín de Porres, en Córdoba, en el cual frente a la indeterminación inicial del espacio libre se planteó su reestructuración a través de una delimitación clara del espacio privado y público. Por un lado, se generaron espacios privados delimitados a través de elementos 'defensivos' (vallas), a la vez que se procedió a la reurbanización de los principales espacios públicos (Morón Serna y Rodríguez Galadí, 2015). Otro ejemplo de reestructuración ha sido el barrio de la Mina, cuya actuación urbana está muy concentrada sobre una franja central de equipamientos públicos, cuya demolición permitió no solo disolver su efecto barrera, sino también introducir un nuevo espacio público con voluntad de traspasar los límites del polígono, capaz de acoger de forma simultánea nuevas parcelas residenciales y equipamientos públicos que den soporte al espacio libre (López de Lucio, 2008). Más allá de calificar el efecto de estas medidas sobre los conjuntos, merece la pena advertir las ventajas que suponen incorporar estas reflexiones en una política integrada, a la par que reconocer las limitaciones de las mismas, sobre todo si se trata de actuaciones sectoriales aisladas.

Sin embargo, otras experiencias internacionales sí han hecho más énfasis en las reflexiones desde el espacio libre, con una valoración diversa sobre el grado de adaptabilidad de sus formas urbanas: desde la consideración del fracaso de la ciudad abierta en los casos de las demoliciones más extensivas; hasta las detecciones de oportunidades de reconfiguración en las estrategias mixtas de remodelación-rehabilitación (García-Pérez et al., 2021). Entre la multitud de experiencias internacionales, este artículo explora la experiencia francesa, cuyas políticas y experiencias de regeneración han sido ampliamente reconocidas como pioneras (Couch et al., 2011).

Los problemas -y primeras actuaciones - en los polígonos franceses son visibles desde la década de los 80. La recesión económica tras los treinta años gloriosos fue más dura en los grands ensembles. De forma acelerada las ventajas del crecimiento autónomo se tradujeron en problemas de aislamiento; la separación de funciones estricta en problemas de movilidad y vitalidad urbana; la utopía del espacio libre en un espacio incontrolado, mal definido y difícil de mantener. La propiedad pública y la tenencia en alquiler estimuló la fuga de los residentes con mayor poder adquisitivo. Los problemas de seguridad y de estigmatización de los ensembles terminaron de construir una espiral de degradación sistémica (Merlin, 2012). Ante esta situación, las primeras medidas llevadas a cabo en el país se enmarcan dentro del programa 'Habitat et vie Sociel, que llevó a cabo actuaciones físicas de carácter superficial y de alcance limitado (Roux et al., 2019). De forma paralela se produjeron las primeras demoliciones de conjuntos, como el paradigmático caso de Les Minguettes (Lyon). Estas experiencias preliminares dieron paso a nuevas fórmulas de desarrollo social y económico, a través de los programas de 'developpement social des quartiers' (desarrollo social de barrios) financiados por la administración central e implementados a nivel local (Wassenberg, 2013). Programas que evolucionarían con el cambio de década y en el marco de un nuevo 'Pacte de relance pour la ville' (programa de estímulo para la ciudad) hacia una nueva aproximación basada en la delimitación y seguimiento de 'zones urbaines sensibles'(barrios vulnerables) y la asociación de las mismas a 'zones de revitalisation urbaine’ (áreas de revitalización urbana) (Couch et al., 2011).

Precisamente en esta dicotomía entre lo físico ('renovation') y lo social ('politique de la ville'), es cuando a partir de 2003 Francia articula una serie de medidas legislativas y presupuestarias que ponen en marcha un gran periodo de renovación de los conjuntos de vivienda (Lelévrier y Melic, 2018). Enfocado en la diversificación social de los polígonos a través de la diferenciación del régimen de tenencia de la vivienda, el Programa Nacional de Renovación Urbana (PNRU) ${ }^{1}$ ha sido objeto de

1 Programme National pour la Rénovation Urbaine (PNRU).

ACE, 16 (4.6) CC BY-ND 3.0 ES | UPC Barcelona, España | Reflexiones en torno a la forma urbana en la experiencia francesa de renovación urbana de los polígonos de vivienda masiva. DOI: http://dx.doi.org/10.5821/ace.16.46.9876 
opiniones divergentes. No se duda de la necesidad de transformación de estos conjuntos. Tampoco de su necesidad de apertura e integración física y mental en la ciudad (Roux et al., 2019). En el centro del debate se sitúa la correlación a veces simplista entre problemas socioeconómicos y la renovación física propuesta - entendida como demolición y construcción de nuevas viviendas-, en muchas ocasiones no justificada (Druot et al., 2007), o con efectos traumáticos sobre la población o no por sí solo efectivos (Gautier et al., 2008; Merlin, 2012). En cualquier caso, la experiencia de regeneración francesa también ha permitido operaciones más allá de la renovación, favoreciendo actuaciones mixtas de reparcelación y rehabilitación (Castrillo, 2010).

En este marco, el objetivo de este artículo es contrastar en qué medida la atención por los espacios libres ha sido objeto de reflexión en las operaciones de regeneración francesas, para además comprobar si dichas actuaciones han tenido un efecto positivo - en términos morfológicos - sobre la calidad urbana de los mismos. Sin olvidar las especificidades de cada contexto, comprender los puntos fuertes y débiles de la estrategia de regeneración francesa permitirá aportar ideas a la práctica española. Para ello, la investigación aplica la metodología de diagnóstico UR-Hesp sobre algunos casos de la región de île-de-France, para contribuir a vislumbrar aciertos y errores que puedan servir de ayuda en las futuras estrategias de regeneración urbana integrada en nuestro país.

\section{El uso de la metodología UR-Hesp para el diagnóstico de la evolución de la calidad urbana}

\subsection{Breve presentación de la metodología UR-Hesp}

Para el desarrollo del estudio sistemático y crítico de las operaciones de regeneración urbana analizadas, este artículo ha utilizado la metodología UR-Hesp. Esta metodología tiene como objetivo ofrecer un diagnóstico de la calidad de los espacios libres de los polígonos, a través de una aproximación morfológica y diacrónica a los mismos. El punto de partida de la metodología es el reconocimiento del papel que el diseño urbano juega en la promoción de una ciudad más compacta, vital, segura e inclusiva. Como se discute en profundidad en la presentación de UR-Hesp, la forma urbana de las ciudades genera un soporte físico estimulante al desarrollo de dichas características - sin ser por ello una garantía de éxito-. Para llevar a cabo esta tarea, UR-Hesp trabaja con nueve variables de estudio físicas, que abordan desde la relación del polígono con el resto del tejido urbano, hasta la configuración en detalle del encuentro de la edificación con el plano del suelo. La explicación en detalle de la citada metodología excede a este artículo, aunque puede consultarse en algunos textos publicados recientemente (García-Pérez et al., 2020).

De entre todas las variables de estudio que incluye UR-Hesp, el artículo focaliza sobre aquellas relacionadas con los procesos de transformación internos al polígono. Cuestiones como los cambios sobre la edificación y la parcelación son sistematizados, gracias al uso de criterios cartográficos homogéneos. De forma complementaria al desarrollo cartográfico, se cuantifican datos (ocupación, edificabilidad, altura media y amplitud) que ayudan a comprender mejor no solo el estado inicial de los polígonos, sino también la incidencia de su transformación. Es precisamente esta combinación de análisis cuantitativo y cualitativo la que permite realizar un análisis crítico de cada una de las operaciones seleccionadas.

Esta parte del trabajo ha sido posible gracias a una adaptación de las fuentes de información al contexto francés (Institut Géographique National, 2017, 2020). Las diferencias en el acceso y presentación de la información geoespacial no han impedido aplicar UR-Hesp al contexto francés, aunque en algunos casos, sí ha sido necesario realizar adaptaciones.

ACE, 16 (4.6) CC BY-ND 3.0 ES | UPC Barcelona, España | Reflexiones en torno a la forma urbana en la experiencia francesa de renovación urbana de los polígonos de vivienda masiva. DOI: http://dx.doi.org/10.5821/ace.16.46.9876 


\subsection{Casos de estudio}

Tanto la figura 1 como la Tabla 1 presentan los casos de estudio seleccionados. La investigación parte de una muestra amplia de casos de estudio, reconocidos en la literatura como paradigmáticos del periodo de construcción de polígonos, no tanto por ser siempre modelos elogiados por la crítica arquitectónica, sino más bien por ser representativos del urbanismo real del periodo de posguerra (Direction générale des patrimoines, 2011). Entre esos 108 casos de estudio se ha realizado una selección de acuerdo con criterios geográficos, dimensionales y de representatividad detallados a continuación:

- En primer lugar, es pertinente centrar la atención sobre la región de île-de-France al ser una de las áreas de mayor desarrollo de polígonos de vivienda masiva durante el periodo de posguerra. La región concentra el 43\% de producción de vivienda masiva², localizados de forma predominante sobre áreas periféricas de la ciudad de París (Lelévrier y Melic, 2018).

- En segundo lugar, teniendo en cuenta criterios dimensionales, este trabajo centra su atención en los desarrollos de vivienda masiva. Aunque no es fácil delimitar cuantitativamente esta cualidad, otros países han asociado este modelo a los desarrollos de más de 1.000 viviendas (Turkington et al., 2004).

- En tercer lugar, la muestra seleccionada incluye polígonos que hayan sido objeto de operaciones de regeneración intensas, financiadas en el marco del PNRU, o de mecanismos autonómicos o público-privados que persiguieran los mismos objetivos. Dichas operaciones se encuentran en una fase muy avanzada, aunque dada la complejidad de las mismas ninguna de ellas ha concluido totalmente.

- Por último, se ha buscado un equilibrio entre localizaciones de cada uno de los polígonos, para evitar el sesgo de la segregación socio-espacial (Figura 1). También se han aplicado otros criterios de diversidad en la muestra, como el valor patrimonial o la diferente propiedad del conjunto. Esta diversidad contribuye a eliminar sesgos en la muestra seleccionada.

Entre todos ellos, finalmente se han seleccionado tres casos de estudio, representativos para comprobar la atención específica de la experiencia francesa en la transformación física de los espacios libres, sus posibles mejoras de calidad urbana, y la discusión de algunos aciertos y errores que pudieran ayudar a la experiencia española de regeneración urbana de polígonos de vivienda masiva.

\section{Transformaciones morfológicas en tres grands ensembles de la región île-de-France}

Esta sección expone los casos de estudio seleccionados, describe los principales objetivos que perseguía su regeneración urbana y presenta el análisis crítico tras la aplicación de la metodología UR-Hesp (Figuras 2-4, Tabla 2).

\subsection{Transformación interior: Les Sorbiers, La Saussaie}

Obra de Jean Fayeton, el conjunto de les Sorbiers - la Saussaie, construido entre 1955 y 1961, se sitúa al sur de París, en el municipio 3 de Chevilly-Larue. Con una extensión de 21 ha contó en su origen con 1.469 viviendas. La composición urbana inicial parte de dos ideas diferenciadas. Por un lado, el conjunto de les Sorbiers, situado al oeste, en el que la edificación de bloques de hasta 5 plantas se

\footnotetext{
2 Las cifras hacen referencia solo a la vivienda social construida durante el periodo 1953-1973.

3 Utilizamos la palabra 'municipal' como estructura administrativa similar al comune francés.
} 


\section{ACE Architecture, City and Environment}

organiza en torno a dos plazas de gran tamaño articuladas a través de una torre, con cierta influencia de la tipología clásica del Palacio Real. Por otro lado, la Saussaie, responde a un esquema de parque inglés de torres en el parque. La edificación, autónoma, define un espacio libre isótropo, de escurridiza fluidez no tanto por su definición (sobre todo en les Sorbiers) sino por su escala (concatenación de plazas de 370×70 y 150×100 metros) (Direction générale des patrimoines, 2011).

Tabla 1. Síntesis de las principales características de los casos de estudio

\begin{tabular}{|l|l|l|l|}
\hline & Les Sorbiers, La Saussaie & Cité des Courtillières & Les Hautes-noues \\
\hline Año de construcción & 1955 -1961 & $1956-1965$ & $1965-1973$ \\
\hline Localización & $\begin{array}{l}\text { Chevilly-Larue } \\
\text { (Val-de-Marne) }\end{array}$ & $\begin{array}{l}\text { Pantin } \\
\text { (Seine-Saint-Denis) }\end{array}$ & $\begin{array}{l}\text { Villiers-sur-Marne } \\
\text { (Val-de-Marne) }\end{array}$ \\
\hline Arquitecto & Jean Fayeton & Émile Aillaud & Denis Honegger \\
\hline $\begin{array}{l}\text { № viviendas (inicial / } \\
\text { actual) }\end{array}$ & $1.469 / 1.636(+11 \%)$ & $1.649 / 1.817(+10 \%)$ & $1.250 / 1.677(+34 \%)$ \\
\hline Programa de rehabilitación & Otros & PNRU & PNRU \\
\hline Periodo de rehabilitación & 2010 - actualidad & $2006-$ actualidad & 2009 - actualidad \\
\hline
\end{tabular}

Fuente: Elaboración propia.

Figura 1. Localización y ortofoto histórica y actual de los casos de estudio seleccionados

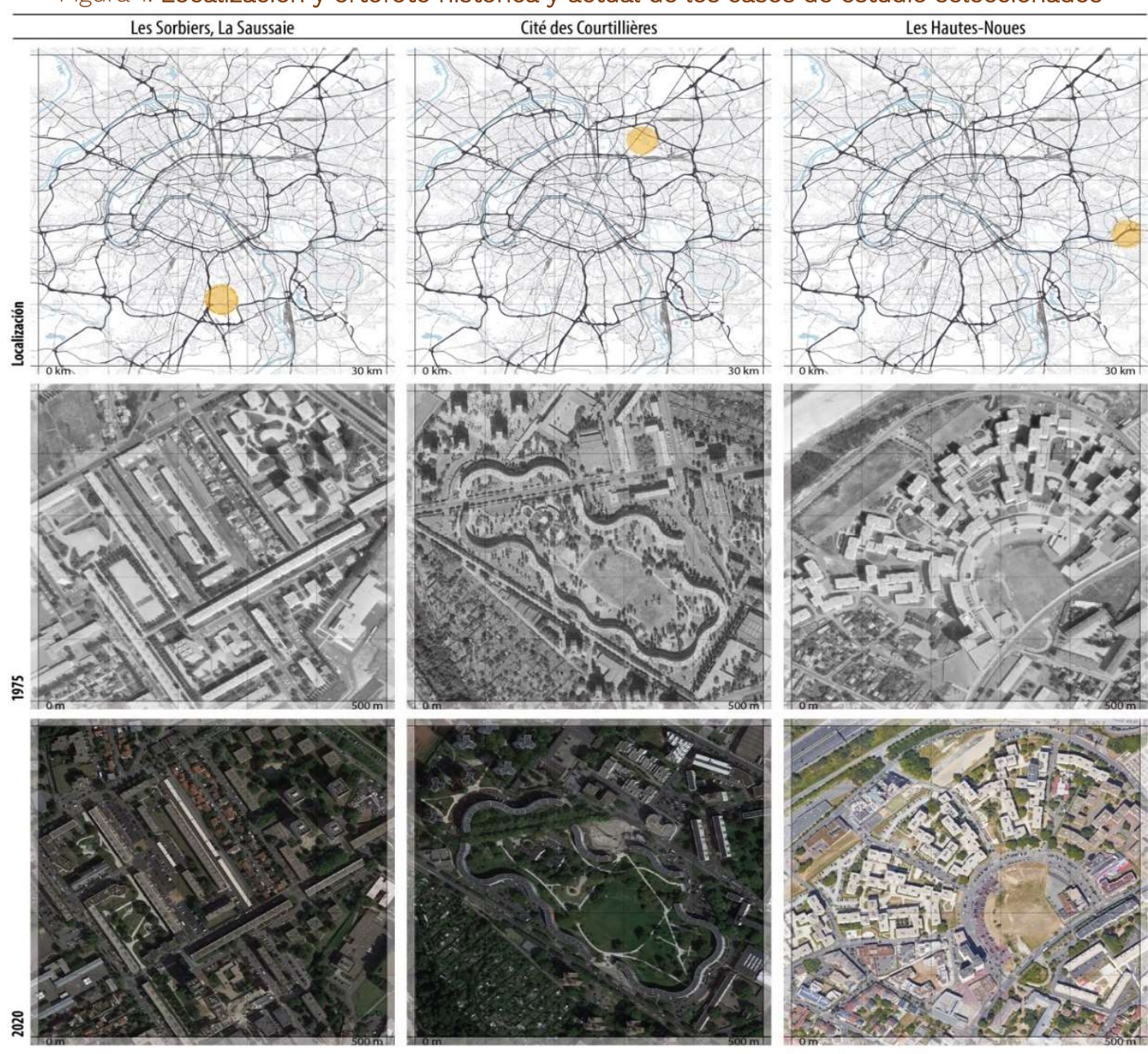

Fuente: elaboración propia a partir de Open Street Maps, Google Earth e Institut Géographique National (IGN).

ACE, 16 (4.6) CC BY-ND 3.0 ES | UPC Barcelona, España | Reflexiones en torno a la forma urbana en la experiencia 
A pesar de que el conjunto no ha sido clasificado como un área vulnerable4, el conjunto sí había perdido atractivo urbano (Nika y Cattin, 2017). Consciente de la situación, el Ayuntamiento de ChevillyLarue planteó un modelo de gestión público-privado llevada a cabo por el grupo Valophis basado en la densificación condicionada a la rehabilitación y remodelación del conjunto. El proyecto, pretende abrir el barrio al resto del municipio, aprovechando también las transformaciones recientes y futuras que a escala metropolitana comienzan a producirse (llegada en 2013 de la nueva línea de tranvía y ampliación de una nueva línea de metro prevista para 2030).

La transformación física se ha efectuado sobre todo sobre sobre el área de les Sorbiers. La operación transforma las grandes manzanas del conjunto, con edificación en bloque de hasta 300 metros, a un nuevo grano urbano más fino. Esta operación permite de forma simultánea:

- Remodelar el sistema de espacios libres existente. El nuevo sistema apuesta por la 'calidad' frente a la 'cantidad', privatizando gran parte del espacio libre. Este ajuste de cantidad permite concentrar el espacio público en espacios concretos, sobre todo en torno al eje de la rue du Berry.

- Introducir nuevas edificaciones residenciales que contribuyen a, en primer lugar, definir mejor la transición entre el espacio libre y privado; en segundo lugar, ampliar y actualizar la oferta tipológica del mercado de la vivienda, no solo en términos espaciales sino también de gestión (vivienda libre / social en alquiler y propiedad); y por último, favorecer la viabilidad económica de la operación, supeditando los nuevos aprovechamientos a la rehabilitación y remodelación del tejido existente.

- Llevar a cabo una demolición selectiva (80 viviendas) de los bloques de mayor longitud, para favorecer la permeabilidad entre los grandes edificios residenciales.

- Rediseñar la estructura y jerarquía viaria para conseguir, por un lado, permeabilizar el tejido y configurarlo con un nuevo grano más fino, y por otro, redistribuir y mejorar las condiciones de estacionamiento en el conjunto.

El análisis crítico tras aplicar la metodología UR-Hesp permite comprobar como:

- El conjunto tenía una gran capacidad de densificación, según revelaba la calidad inicial (pobre). La renovación y el aumento de nueva vivienda (249 en total) ha permitido mejorar de forma ligera las condiciones de densidad física hacia formas urbanas más compactas e intensas. Los nuevos desarrollos no han incrementado la altura media del conjunto, demostrando una atención por la preexistencia a la vez que se humanizan las dimensiones de los espacios libres. Sin embargo, el esfuerzo de esta intervención se ha centrado sobre les Sorbiers, existiendo todavía margen de mejora en la Saussaie.

- La nueva edificación junto a la reparcelación propuesta en les Sorbiers ha permitido mejorar cuantitativamente la relación entre el espacio construido y libre desde valores medios a altos, con una mejora del 32\% respecto a la situación previa.

- Sin embargo, la diversidad funcional apenas ha mejorado (niveles bajos). De forma crítica, se ha desaprovechado la oportunidad de generar ejes multifuncionales, de mayor vitalidad urbana, a la vez que se concentraba el espacio libre de uso público sobre ejes concretos (rue du Berry).

En definitiva, la remodelación aplicada sobre el entorno de les Sorbiers presenta mejoras sustanciales en la búsqueda de niveles de densidad que favorezcan la vitalidad de los espacios libres, a su vez concentrados en torno a nuevos espacios de centralidad que, sin embargo, no han terminado de alcanzar su máximo potencial al aplicar criterios de diversificación funcional.

\footnotetext{
${ }^{4}$ La correspondencia francesa a las áreas vulnerables son las denominadas Zones Urbaines Sensibles (ZUS).
}

ACE, 16 (4.6) CC BY-ND 3.0 ES | UPC Barcelona, España | Reflexiones en torno a la forma urbana en la experiencia francesa de renovación urbana de los polígonos de vivienda masiva. DOI: http://dx.doi.org/10.5821/ace.16.46.9876 
Tabla 2. Indicadores de densidad

\begin{tabular}{|c|c|c|c|c|c|c|}
\hline & \multicolumn{2}{|c|}{ Les Sorbiers, La Saussaie } & \multicolumn{2}{|c|}{ Cité des Courtillières } & \multicolumn{2}{|c|}{ Les Hautes-Noues } \\
\hline & 1975 & 2015 & 1975 & 2015 & 1975 & 2015 \\
\hline Ocupación & $22 \%$ & $25 \%$ & $18 \%$ & $19 \%$ & $36 \%$ & $28 \%$ \\
\hline Edificabilidad & 1,19 & 1,22 & 0,87 & 0,97 & 1,49 & 1,33 \\
\hline Altura media & 5,34 & 4,87 & 4,91 & 5,20 & 4,08 & 4,76 \\
\hline Amplitud & 0,65 & 0,61 & 0,94 & 0,83 & 0,43 & 0,54 \\
\hline Amplitud corregida & 0,60 & 0,40 & 0,83 & 0,73 & 0,31 & 0,21 \\
\hline $\begin{array}{l}\text { Valoración de } \\
\text { densidad final }\end{array}$ & Pobre & Estándar & Pobre & Pobre & Estándar & Estándar \\
\hline
\end{tabular}

Fuente: Elaboración propia.

\subsection{Conservación y actualización: Cités des Courtillières}

Cités des Courtilières se sitúa al norte de París, en el municipio de Pantin. Este grand ensemble fue pionero por varias razones: la primera, por ser impulsor de la urbanización de la zona, al construirse sobre unos terrenos vacantes de unas 26 ha, próximos al fuerte de Aubervilliers. La segunda, por las técnicas constructivas utilizadas, al introducir la prefabricación en el debate de vivienda social de posguerra. Y, por último, por tratarse de una de las primeras operaciones de vivienda social del arquitecto E. Aillaud, en la que optó por una solución formal de ciudad parque (Lefrançois y Landauer, 2011, pp. 52-79). El conjunto cuenta con un elemento central conocido como serpentin, una barra sinuosa que configura un espacio libre introvertido (también denominada pelouse) ${ }^{5}$, al que se adhieren otros grupos de torres-trípode y bloques intercalados con los equipamientos que promueven la autosuficiencia funcional del conjunto, idea dominante en la teoría urbanística del periodo.

Gracias a estas características pioneras, y también debido en parte a los intentos de derribo del conjunto durante el principio de siglo, Courtilliéres ha sido uno de los primeros grand ensembles en recibir la etiqueta 'patrimonio del siglo XX', reconociendo el valor cultural del conjunto (Díez de Pablo y Moya González, 2016). Sin embargo, el proceso de patrimonialización no podía obviar la necesidad de actualización del conjunto, que para aquel entonces ya había comenzado a experimentar procesos de concentración de población vulnerable considerables, habiendo sido incluido en las políticas estatales como área de actuación de regeneración prioritaria6.

El reto de la operación pasaba por hacer compatible la transformación —-focalizada en la renovación de tejidos selectiva - y la conservación —basada en la rehabilitación y reurbanización extensiva del conjunto-. El plan, obra del despacho l'AUC proponía (Klouche, 2007):

- Compatibilizar la patrimonialización con la transformación, reforzando el serpentin y su pelouse, realizando operaciones sutiles que permitieran mejorar sus accesos y su hábitat, al desenclavar el espacio central para convertirlo en un parque abierto sin perder su carácter introvertido.

- Introducir nuevos modelos espaciales y de gestión de vivienda bien sea por demolición de viviendas existentes o por la modificación de usos de grandes terrenos dotacionales. En el primer caso, destaca la operación de renovación del área de bloques situada al norte del serpentin. El segundo, todavía en fase de ejecución, contempla la transformación de los terrenos deportivos al noroeste del conjunto.

\footnotetext{
${ }^{5}$ En este texto, el término pelouse (cuya traducción literal es césped) hace referencia a una superficie verde y ajardinada. ${ }^{6}$ En francés denominado quartier prioritarie (QP), incluidos en la politique de la ville.
}

ACE, 16 (16) CC BY-ND 3.0 ES | UPC Barcelona, España | Reflexiones en torno a la forma urbana en la experiencia francesa de renovación urbana de los polígonos de vivienda masiva. DOI: http://dx.doi.org/10.5821/ace.16.46.9876 
- Abrir el conjunto al resto del tejido urbano, al introducir nuevos equipamientos de rango municipal, que sirvan de charnela entre el corazón del conjunto y su pelouse.

- De forma transversal a todas las actuaciones anteriores se ha producido una reurbanización de la totalidad del entorno, cuya calidad ha merecido el reconocimiento del Centro de Cultura Contemporánea de Barcelona, siendo finalista del Premio Europeo del Espacio Público Urbano (Navas, 2019).

De acuerdo con la metodología UR-Hesp, podemos indicar que:

- La diversificación en el hábitat no ha producido todavía cambios en el análisis cuantitativo de densidad (en parte porque la operación todavía no está finalizada). Sin embargo, el barrio contaba con grandes oportunidades para la misma (baja ocupación y baja edificabilidad). Es predecible que los efectos de la densificación se presenten polarizados en el conjunto, entre los espacios vacantes de fácil remodelación al exterior del polígono, y de forma selectiva al interior del mismo.

- La nueva edificación residencial ha introducido nuevos usos terciarios en planta baja, sobre todo en torno a los ejes principales que la operación de regeneración urbana tiende a potenciar.

- El trabajo sobre los accesos a la pelouse, de forma intuitiva parecen mejorar la situación del parque central. Más allá de la mejora sustancial de la reurbanización, la operación ha obviado algunas cuestiones de diseño urbano que quizá hubiera podido explorar, como la transformación hacia un parque equipado, o el estudio de accesos de la edificación que hubiera multiplicado la facilidad de acceso al parque.

En este caso, frente a la estrategia de privatización del espacio libre, la principal novedad de la intervención es la transformación del espacio central de carácter introvertido al interior del serpentin en un espacio abierto. Esta actuación refuerza los valores patrimoniales con los que el conjunto fue concebido, trasladando las oportunidades de densificación al exterior del conjunto.

\subsection{Densificación sobre el borde urbano: Les Hautes-Noues}

En el municipio de Villiers-sur-Marne, al este de París, les Hautes-Noues representa un segundo periodo de construcción de polígonos (1965-1973), el cual se caracteriza por la superación de los preceptos más rígidos del urbanismo funcionalista. Sobre un área de 20 ha, D. Honegger proyecta un crecimiento autárquico inspirado en los resultados espaciales de los tejidos medievales, sin renunciar a los recursos propios de la modernidad. Les Hautes-Noues es un ejercicio que explora la creación de un sistema de crecimiento flexible, adaptable al terreno y modulable, a través de la agrupación de tipos de viviendas en dirección horizontal y vertical. Calificado como ejercicio utópico, el conjunto se ordena a través de tres sectores diferenciados en torno a una semicircunferencia utilizada como pelouse. El primero de ellos, acogía los usos comerciales, formalizado a través de las ya desaparecidas arcades. El segundo, de carácter residencial, en cuyo diseño D. Honegger procuró restringir el paso del vehículo privado. Sin embargo, pronto la presión del automóvil aumentó sobre el polígono, y la realidad urbana modificó radicalmente el carácter de los espacios libres, casi laberínticos, convirtiéndolos en un gran espacio de estacionamiento al aire libre7 (Texier y Radouan, 2010). El borde exterior -en contacto con la autovía A4, construida de forma casi paralela a la edificación del conjunto-, estaba destinado a usos deportivos, aunque la realidad se impondría convirtiendo la bien intencionada área como un espacio vacío (Direction générale des patrimoines, 2011).

\footnotetext{
7 De hecho, es este 'escenario real' el que se ha considerado en el cartografiado del estado inicial.
} 


\section{ACE Architecture, City and Environment}

Sin embargo, si contrastamos la esperanzadora utopía formal de Honegger con la realidad social del polígono, nos encontramos con un urbanismo real diverso al planificado. Este hecho, constante en muchos de los desarrollos funcionalistas, justificó la necesidad de actuación de regeneración urbana en el conjunto, enmarcada en la política ANRU, cuyo convenio se inicia en el año 2009.

Figura 2. Ordenación interna y transformación morfológica

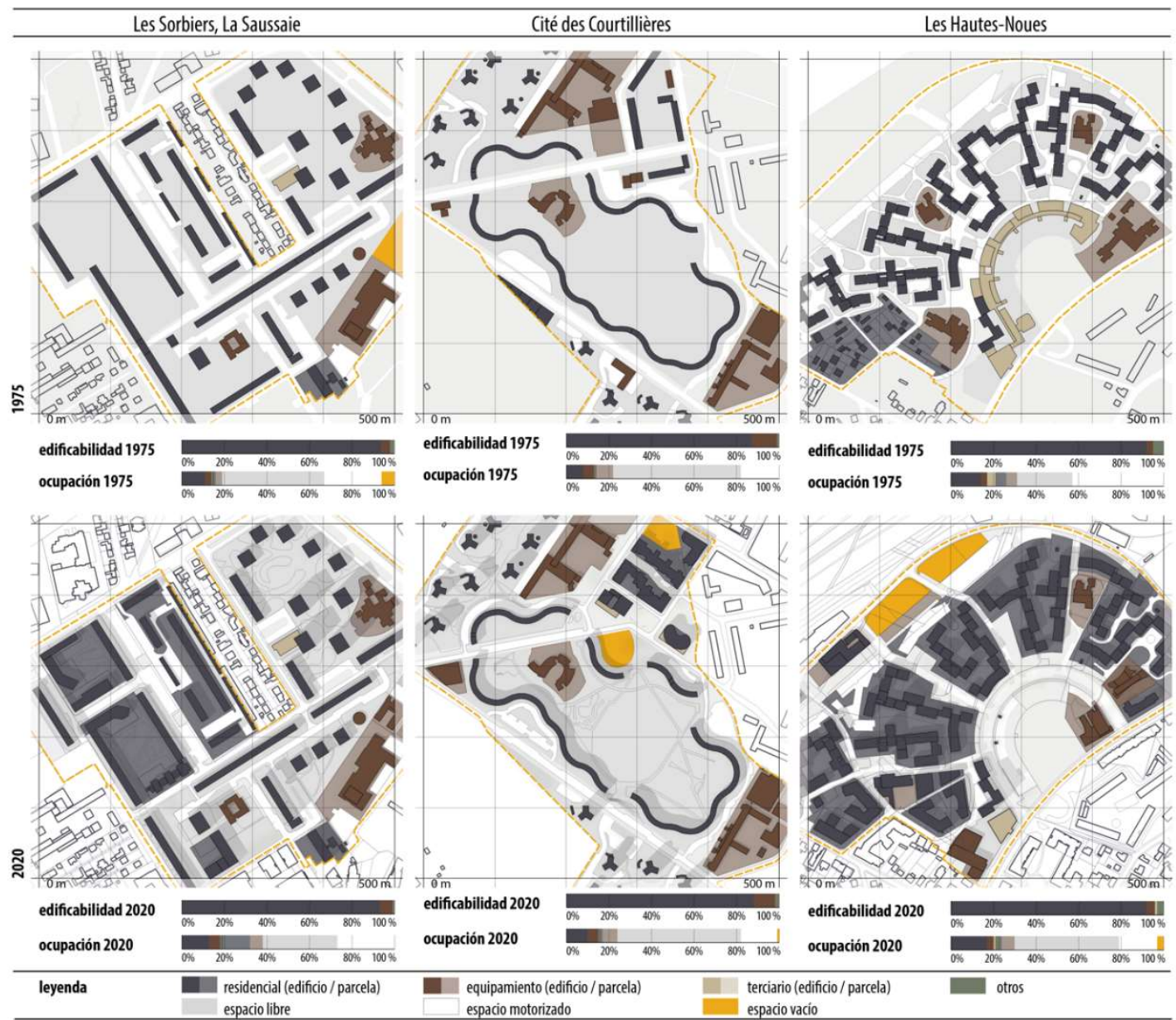

Fuente: elaboración propia a partir de datos del Institut Géographique National (IGN).

Al igual que muchas de las operaciones ANRU, la actuación persigue la diversificación social de los polígonos, a través de un aumento de la diversidad física (Grand Paris Aménagement, 2017). De forma concreta, la transformación se basa en:

- Transformar los espacios de borde del polígono, apostando por su densificación (construcción de 490 viviendas nueva de acceso en propiedad). El aumento de diversidad social y espacial va a producirse por tanto al exterior del polígono (Faure, 2015).

- Rehabilitar la edificación existente (incluyendo una operación de desamiantado), reestructurando también el espacio libre. Para ello, la nueva ordenación introduce una nueva jerarquía viaria (siendo necesario una demolición selectiva de 47 viviendas), que genera una retícula clara entre el borde del polígono y el centro del mismo.

- Una vez generada la nueva estructura viaria, la reparcelación del espacio libre permite una nueva agrupación de la edificación, privatizando el espacio libre entre bloques. Para garantizar 
el uso peatonal del espacio libre (de uso privado), el estacionamiento queda limitado a bolsas en superficie y subterráneas al exterior del conjunto (Ministere de l’Equipement, 2004).

- Mejorar los servicios del polígono, a través de un proceso de dotación que incluye hasta cuatro nuevos equipamientos (previa demolición de los más obsoletos).

El estudio crítico bajo la metodología UR-Hesp, permite reflexionar sobre las siguientes cuestiones:

- Las oportunidades de densificación del grand ensemble eran medias, al tener una calidad inicial en términos de densidad 'estándar'. La situación específica característica del espacio libre no cualificado en los bordes del polígono ha permitido introducir nuevas tipologías residenciales con una muy baja necesidad de demolición. Aunque en términos netos ocupación y edificabilidad han disminuido (aunque sin perder su condición de calidad 'estándar'), una mirada en detalle revela un efecto positivo, ya que al observar de forma paralela los valores brutos, se ha transformado sobre todo suelo destinado al estacionamiento en espacios libres de diseño orientado al peatón.

- Esta operación de reubicación del estacionamiento se ha acompañado de una operación de reparcelación, que ha ratificado la clarificación del uso y propiedad de los espacios libres. La nueva organización en manzanas ha permitido, en términos cuantitativos y cualitativos reequilibrar la relación de espacio libre frente al construido, desde valores altos (resultado influenciado por el sobreuso del espacio libre como espacio de estacionamiento) a muy altos (tras la transformación de espacio de estacionamiento en libre de uso peatonal). Además, esta operación perseguía la creación de pequeños espacios colectivos en los principales accesos a las manzanas residenciales o dotacionales, y la consolidación de la pelouse como gran espacio central de convivencia. Frente a las primeras, que ya han entrado en carga, la operación de remodelación e intensificación sobre el vacío central todavía queda pendiente.

- A pesar de la mejora de la oferta de equipamientos públicos, precisamente la diversificación funcional y su oportunidad en entornos críticos como la pelouse es uno de los puntos mejorables del proyecto de regeneración.

Aunque la actuación principal de densificación sobre el borde urbano se acompaña de una remodelación profunda del sistema de espacios libres, quizá el punto más crítico de la propuesta sea las implicaciones sociológicas que tiene la disposición de los nuevos edificios residenciales (Lelévrier, 2013). El tiempo confirmará las ventajas y desventajas de la operación, cuyos efectos en la actualidad todavía no pueden evaluarse.

\section{Luces y sombras de la experiencia de renovación francesa}

Esta comunicación ha explorado la transformación morfológica de algunos ejemplos de regeneración urbana de polígonos de vivienda masiva en la región de île-de-France. Gracias al uso de la metodología UR-Hesp - convenientemente adaptada al contexto francés-, se ha evaluado la calidad urbana en términos de densidad, diversidad funcional y configuración del espacio intermedio, a través de una aproximación cuantitativa y cualitativa. La metodología aplicada se nutre no solo de datos, sino también de cartografías analíticas, facilitando la interpretación y comparación de los resultados (figuras 2-4).

Como ha podido discutirse, la máxima que persigue la experiencia francesa de regeneración de polígonos es la diversificación residencial en términos formales y de tenencia que permita en un escenario a corto, medio y largo plazo desenclavar socialmente estos conjuntos urbanos. Las experiencias analizadas han dado forma a ese objetivo desde diferentes aproximaciones, aprovechando las oportunidades específicas de cada entorno.

ACE, 16 (4.6) CC BY-ND 3.0 ES | UPC Barcelona, España | Reflexiones en torno a la forma urbana en la experiencia francesa de renovación urbana de los polígonos de vivienda masiva. DOI: http://dx.doi.org/10.5821/ace.16.46.9876 


\section{ACE Architecture, City and Environment}

Figura 3. Evolución de la estructura parcelaria

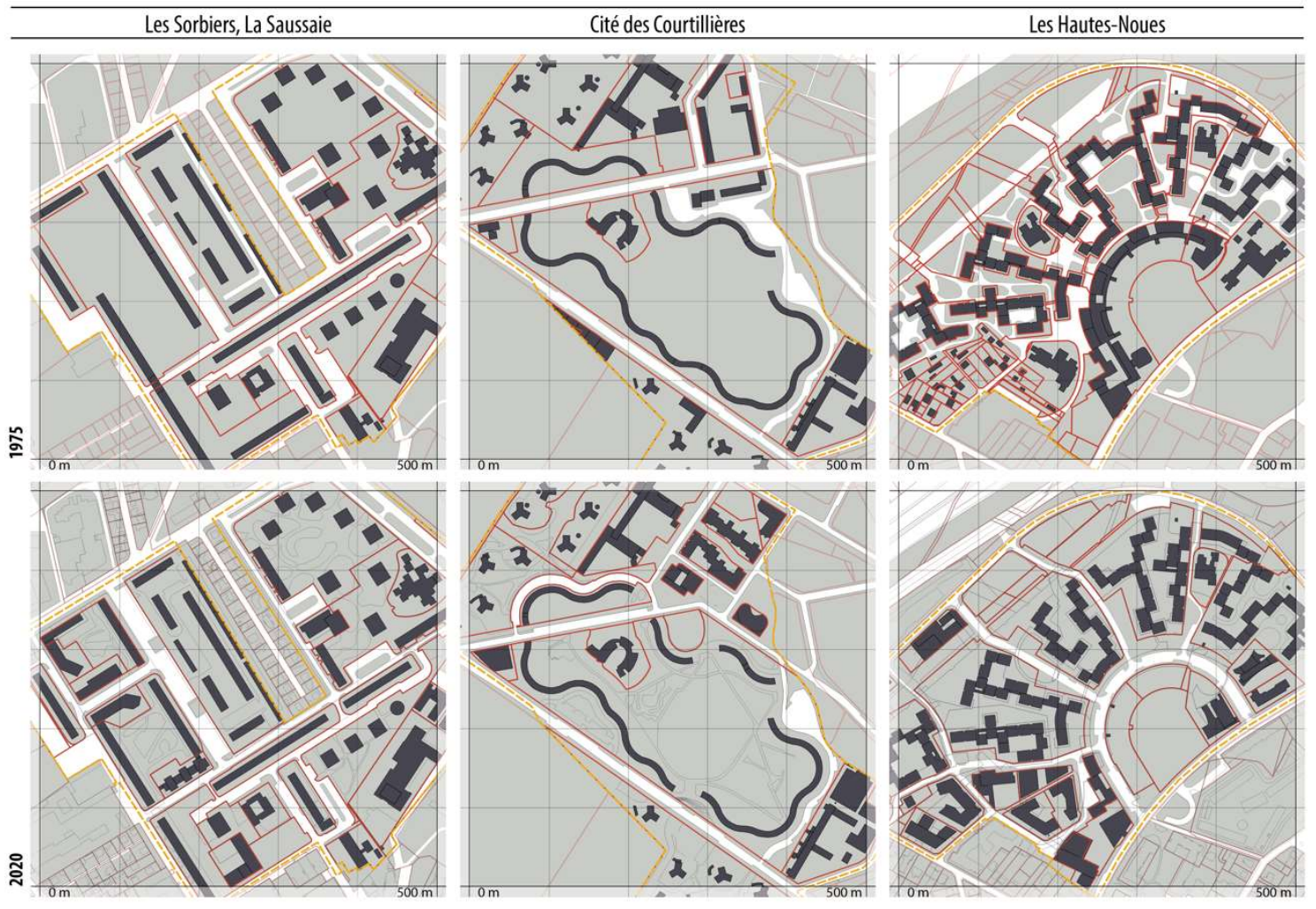

Fuente: elaboración propia a partir de datos del Institut Géographique National (IGN).

Una visión conjunta sobre los casos de estudio analizados reconoce el efecto positivo que supone modificar la escala de trabajo, del edificio a la dimensión urbana. Desde esta nueva perspectiva, y gracias a la aplicación de UR-Hesp, ha podido comprobarse el margen de mejora que tenían los polígonos de vivienda aceptando procesos de densificación, diversificación funcional y reconfiguración de los espacios libres. En concreto:

- Los procesos de densificación han sido más comunes en los casos de estudio analizados, aunque su formalización no ha sido homogénea (densificación sobre suelo vacante al exterior o al interior del polígono). Estos resultados preliminares deberán ser contrastados en el tiempo con otros estudios que desde el ámbito de la sociología comienzan a advertir que la solución formal adoptada podría condicionar la coexistencia entre los nuevos y antiguos residentes, más que su integración (Lelévrier, 2013).

- Menos afortunadas han sido las oportunidades de diversificación funcional. A pesar de los esfuerzos por mejorar la dotación de los polígonos, los incrementos - al menos en términos cuantitativos - son pequeños y siguen lejos de niveles de calidad que permitan desarrollar entornos urbanos más intensos. Además, en términos cualitativos los ligeros procesos de diversificación en muchos casos no se han producido sobre entornos prioritarios (ej: potenciación de un nuevo eje o área de proximidad, etc.).

- En cuanto a la reconfiguración de los espacios libres, las actuaciones de reparcelación han contribuido, por un lado, a disolver los problemas de gestión y mantenimiento asociados a la falta de claridad parcelaria, y por otro, han contribuido a ajustar la relación entre cantidad de espacio libre y construido. Sin embargo, será necesario terminar de aplicar UR-Hesp, sobre todo para comprobar la calidad del diseño 'a la altura de los ojos'. Como avanzan 
algunas investigaciones, en ocasiones la configuración de los nuevos límites, unido a la menor atención por la diversificación funcional, puede contribuir a reestablecer más bien un espacio libre defensivo (Newman, 1972), con resultados formales característicos del urbanismo 'ordinario', menos orientado hacia su dimensión pública (Manzano Gómez, 2017).

Además, los resultados preliminares de esta investigación parecen indicar que, frente a la idea de demolición extensiva que caracteriza la crítica a la experiencia de regeneración francesa, el marco político que favorece actuaciones de renovación, reparcelación y rehabilitación es flexible y adaptable a las necesidades y oportunidades específicas de cada polígono. Al respecto, parece interesante abrir una línea de investigación tras esta primera aproximación. Futuros estudios deberían profundizar en la responsabilidad que puede tener no solo la política, sino también el proyecto de regeneración en los aciertos y errores de la experiencia francesa.

El análisis de la experiencia francesa permite extrapolar algunas ideas que pueden ser útiles en la definición de políticas y estrategias españolas. La idea de densificación y reparcelación, tan extendida en el caso francés, está permitiendo transformar en términos morfológicos la ciudad abierta del movimiento moderno. Aunque los resultados pueden ser desiguales, el hecho de superar la visión 'edificatoria' de la experiencia española es un paso importante. Si realmente se adopta la necesidad de resolver los problemas específicos de los conjuntos a corto, medio y largo plazo, no podemos repetir el error de confiar la solución en una ordenación fundada, en muchas ocasiones, tan solo desde la edificación.

Aunque existen diferencias administrativas que dificultan una transferencia directa de las ideas de reparcelación y densificación de los polígonos, sí parecería interesante explorar soluciones de gestión innovadoras adaptadas al contexto español que permitieran pensar la regeneración de los conjuntos desde una aproximación mixta urbano-edificatoria. Por supuesto, teniendo en cuenta las primeras advertencias que desde ámbitos como la sociología están comenzado a surgir al evaluar la experiencia francesa. ¿Cómo podemos reconfigurar un espacio libre que en muchas ocasiones no fue pensado como tal, trabajando desde las preexistencias? Quizá esa sea una de las lecciones más valiosas de la experiencia francesa (Panerai y Mangin, 2002, p. 83), que sin duda deberemos adaptar a la realidad específica de nuestro país.

Sin embargo, esta investigación no está exenta de límites. Por un lado, el artículo es una aproximación preliminar que deberá completarse con el análisis total de las variables de UR-Hesp. Confirmar los efectos físicos de la regeneración a escala de ciudad (sobre todo en términos de integración y relación tanto con la ciudad como con el entorno inmediato), y a nivel de detalle complementará la información aquí aportada, y contribuirá a matizar los resultados presentados. Por otro lado, existen otras limitaciones, propias de UR-Hesp, derivadas no solo del enfoque físico-morfológico de la aproximación y su necesidad de contraste desde otras dimensiones socioeconómicas, sino también de la propia metodología empleada, que han sido discutidas en textos previos (García-Pérez et al., 2020).

A pesar de las limitaciones, este enfoque sí ha permitido realizar un análisis crítico preliminar de las principales transformaciones morfológicas en algunos casos de estudio de la región de île-de-France, y su incidencia en la calidad urbana de los espacios libres, contribuyendo a aportar nuevas ideas al complejo debate de la regeneración de polígonos de vivienda masiva.

ACE, 16 (4.6) CC BY-ND 3.0 ES | UPC Barcelona, España | Reflexiones en torno a la forma urbana en la experiencia francesa de renovación urbana de los polígonos de vivienda masiva. DOI: http://dx.doi.org/10.5821/ace.16.46.9876 


\section{ACE Architecture, City and Environment}

Figura 4. Remodelación de la edificación y la parcelación

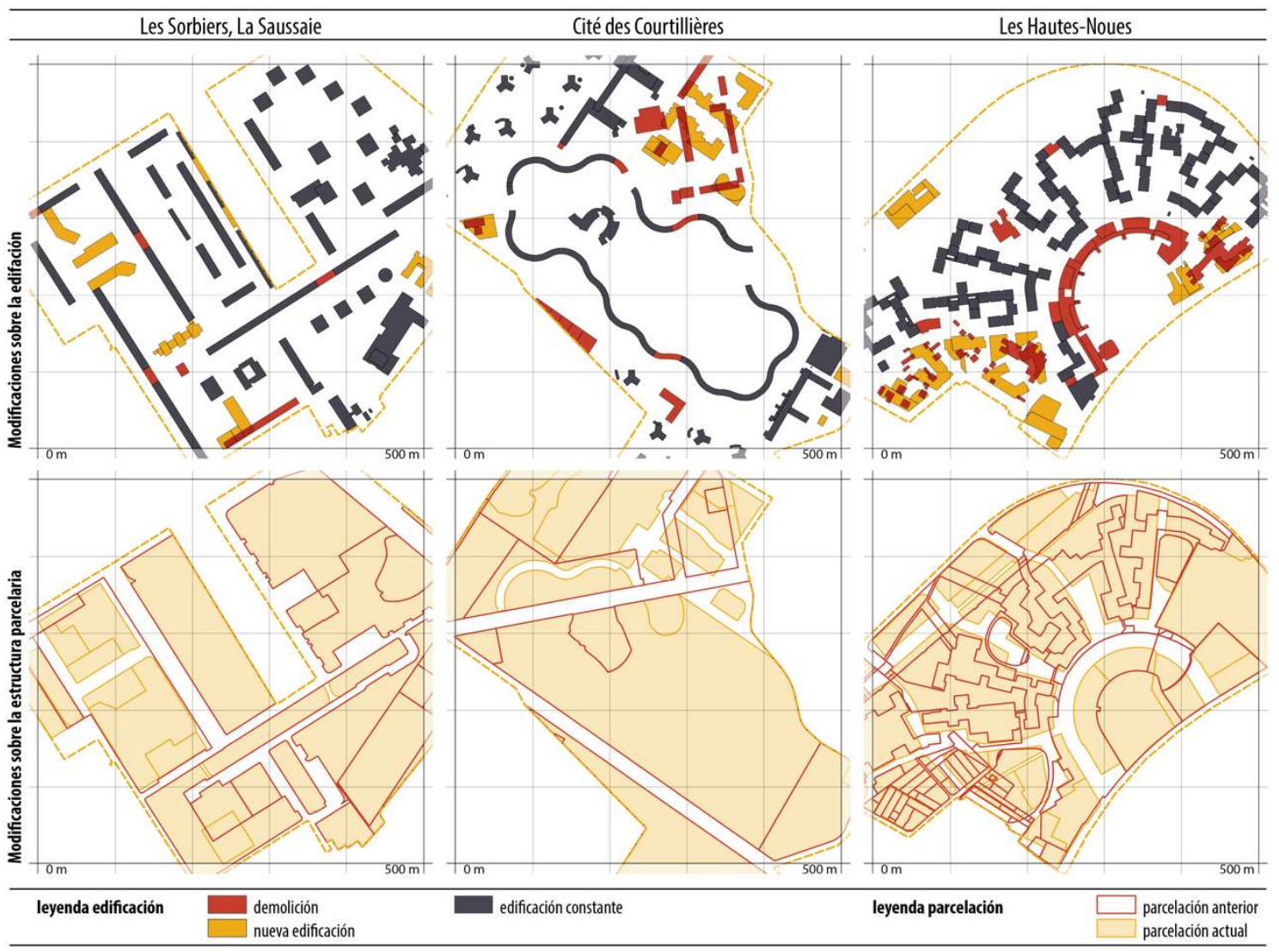

Fuente: elaboración propia a partir de datos del Institut Géographique National (IGN).

\section{Agradecimientos}

Este trabajo se enmarca en la estancia predoctoral que el autor realizó durante los meses de septiembre a octubre del año 2019 en la Université Paris 1 Panthéon-Sorbonne. La estancia ha sido financiada por el Ministerio de Economía y Competivididad en el marco de la ayuda predoctoral BES2015-072536, y forma parte del proyecto UR-Hesp (BIA2014-60059-R). También por el grupo de investigación de referencia PUPC (Paisajes Urbanos y Proyecto Contemporáneo) financiado por el Gobierno de Aragón (T44_20R). El autor quisiera agradecer a P. Petsimeris, catedrático de la UFR de Géographie, su supervisión y orientación para la realización del trabajo. También a J.I. Vila Vázquez, por su inestimable ayuda en la consulta, obtención y tratamiento de bases de tatos y cartografía. También al Colegio de España de la Ciudad Internacional Universitaria de París y a sus residentes, por facilitar la realización de la estancia.

Conflicto de intereses: El autor declara que no hay conflicto de intereses. 


\section{Bibliografía}

Castrillo, M. (2010). El urbanismo de renovación de grandes conjuntos de vivienda social en Francia, 2004-2008. Proyecto, Progreso, Arquitectura, 2, 54-67. DOI: https://doi.org/10.12795/ppa.2013.i2.05

Castrillo Romón, M. A., Fernández-Maroto, M., y Jiménez Jiménez, M. (2016). Espacios libres del Movimiento Moderno: debates y nuevos instrumentos para el mejoramiento urbano. Huerta del Rey como laboratorio. Bitácora Urbano Territorial, 26(1), $23 . \quad$ DOI: https://doi.org/10.15446/bitacora.v26n1.54626

Cervero Sánchez, N. (2020). La envolvente en la rehabilitación de conjuntos de vivienda protegida. ACE: Architecture, City and Environment, 14(42). DOI: https://doi.org/10.5821/ace.14.42.8960

Cocco, F., y López, F. A. (2015). Ajustes razonables en la rehabilitación de polígonos de viviendas: aplicación al barrio Montserrat de Terrassa (Barcelona). ACE: Architecture, City and Environment, 10(29), 31-58. DOI: https://doi.org/10.5821/ace.11.29.3693

Couch, C., Sykes, O., y Börstinghaus, W. (2011). Thirty years of urban regeneration in Britain, Germany and France: The importance of context and path dependency. Progress in Planning, 75(1), 1-52. DOI: https://doi.org/10.1016/j.progress.2010.12.001

Daumal, F., Monzón, M., y López-Mesa, B. (2018). La necesidad de conciliar la normativa de habitabilidad con la rehabilitación acústica de la vivienda mínima. Informes de la Construcción, 70(549), 241. DOI: https://doi.org/10.3989/id.56696

Díaz Gómez, C., Cornadó Bardón, C., Vima Grau, S., Ravetllat Mira, P. J., y Garcia-Almirall, M. P. (2019). Intervenciones de rehabilitación en grandes conjuntos habitacionales construidos durante el periodo 1950-1975. ACE: Architecture, City and Environment, 14(41), 11-34. DOI: https://doi.org/10.5821/ace.14.41.6538

Díez de Pablo, A. (2015). La vivienda social como patrimonio urbano. Análisis de la patrimonialización de tres barrios de promoción oficial madrileños [Tesis Doctoral dirigida por Luis Moya]. E.T.S. Arquitectura (UPM).

Díez de Pablo, A., y Moya González, L. (2016). Iniciativas de patrimonialización de conjuntos de vivienda social en Europa. Ciudad y Territorio, Estudios Territoriales, XLVIII (190), 643-654. Recuperado de https://recyt.fecyt.es/index.php/CyTET/article/view/76509

Díez Medina, C. (Ed.). (2015). El legado de la vivienda moderna / Modernist Mass Housing Legacy. ZARCH: Journal of interdisciplinary studies in Architecture and Urbanism, 5.

Díez Medina, C., y Monclús, J. (2020). La ciudad de bloques. Reflexiones retrospectivas y prospectivas sobre los poligonos de vivienda "modernos". Abada Editores.

Díez Medina, C., y Monclús, J. (2017). Dealing with mass housing estates legacy: The need of specific diagnoses from an urban design perspective. Proceedings 24th ISUF 2017 - City and Territory in the Globalization Age, 309-322. DOI: https://doi.org/10.4995/ISUF2017.2017.5887

Direction générale des patrimoines. (2011). Les grands ensembles: une architecture du XXe siècle (É. Henry (Ed.)). Carré.

Druot, F., Lacaton, A., y Vassal, J.-P. (2007). Plus. La vivienda colectiva. Territorio de excepción. Gustavo Gili.

Faure, L. (2015). L'intensification dans les projets de rénovation urbaine en lle de France. Contribution de l'ANRU à l'étude menée par l'Observatoire Régional du Foncier d'île de France (T. Asselin y F. Leonhardt (Eds.)). Agence Nationale pour la Rénovation Urbaine.

García-Pérez, S. (2019). Regeneración urbana de poligonos de vivienda masiva. Criterios para la evaluación y diagnóstico de la calidad de los espacios libres [Tesis Doctoral dirigida por J. Monclús y J. M.a Ezquiaga, tutorizada por C. Díez Medina]. Universidad de Zaragoza.

ACE, 16 (4.6) CC BY-ND 3.0 ES | UPC Barcelona, España | Reflexiones en torno a la forma urbana en la experiencia francesa de renovación urbana de los polígonos de vivienda masiva. DOI: http://dx.doi.org/10.5821/ace.16.46.9876 
García-Pérez, S., Monclús, J., y Díez Medina, C. (2021). Intervention follows diagnosis: the impact of analysis in urban regeneration of open space in mass housing estates. Territorio.

García-Pérez, S., Oliveira, V., Monclús, J., y Díez Medina, C. (2020). UR-Hesp: A methodological approach for a diagnosis on the quality of open spaces in mass housing estates. Cities, 103, 102657. DOI: https://doi.org/10.1016/j.cities.2020.102657

Gautier, J., Piquéras, C., Aris, D., y Henry, E. (Eds.). (2008). Faut-il protéger les grands ensembles? Direction de l'Architecture et du Patrimoine - Ministère de la Culture et de la Communication.

Grand Paris Aménagement. (2017). PRU des Hautes Noues. Villiers-sur-Marne (94).

Higueras García, E. (2015). La vocación del espacio público en bloques abiertos. Propuesta metodológica y caso de estudio en Moratalaz. Planur-e: territorio, urbanismo, paisaje, sostenibilidad y diseño urbano, 4, 1-12. Recuperado de https://www.planur-e.es/articulos/ver/la-vocaci-n-delespacio-p-blico-en-bloques-abiertos

Institut Géographique National. (2017). Géoservices IGN. Recuperado de https://geoservices.ign.fr/documentation/diffusion/index.html

Institut Géographique National. (2020). Remonter les temps. Recuperado de https://remonterletemps.ign.fr/

Klouche, D. (2007). Pour l'urbanisme de recyclage. Urbanisme. Rénovation urbaine: enjeux, mise en oeuvre, qualités, H.S. 30, 17-18.

Lefrançois, D., y Landauer, P. (2011). Émile Aillaud. Infolio, Éd. du Patrimoine.

Lelévrier, C. (2013). Social mix neighbourhood policies and social interaction: The experience of newcomers in three new renewal developments in France. Cities, 35, 409-416. DOI: https://doi.org/10.1016/j.cities.2013.03.003

Lelévrier, C., y Melic, T. (2018). Impoverishment and Social Fragmentation in Housing Estates of the Paris Region, France. En D. B. Hess, T. Tammaru, y M. van Ham (Eds.), Housing Estates in Europe (pp. 313-338). Springer. DOI: https://doi.org/10.1007/978-3-319-92813-5 14

López de Lucio, R. (2008). La Rehabilitación de la ciudad existente. El Plan Especial de reforma y reordenación del barrio de La Mina y documentos complementarios 2000-2006. En Ordenar el territorio, proyectar la ciudad, rehabilitar los tejidos existentes. La relevancia del planeamiento a través de los premios nacionales de urbanismo (pp. 121-165). Ministerio de la Vivienda, Secretaría General Técnica, Centro de Publicaciones.

Manzano Gómez, N. A. (2017). Espacio público en una periferia de París: Vitry-sur-Seine. Ciudades, 17, 201. DOI: https://doi.org/10.24197/ciudades.17.2014.201-218

Martínez Gutiérrez, A. (2017). La renovación de los polígonos de Barcelona [Trabajo fin de máster dirigido por Francisco Peremiquel Lluch]. Universitat Politècnica de Catalunya.

Merlin, P. (2012). Des grands ensembles aux cités: l'avenir d'une utopie. Ellipses.

Ministere de l'Equipement. (2004). Stationnement et rénovation urbaine. Problématique, études de cas. CDC.

Monclús, J., y Díez Medina, C. (2016). Modernist housing estates in European cities of the Western and Eastern Blocs. Planning Perspectives, 314), 533-562. DOI: https://doi.org/10.1080/02665433.2015.1102642

Morón Serna, E., y Rodríguez Galadí, J. I. (2015). Rehabilitación integral de barriadas con dificultades sociales en Andalucía. La experiencia de San Martín de Porres en Córdoba. Informes de la Construcción, 67(Extra-1), m027. DOI: https://doi.org/10.3989/ic.14.051

Navas, T. (2019). Mejjora integral de los espacios libres de Les Courtillières. Publicspace. Recuperado de https://www.publicspace.org/es/obras/-/project/k243-les-courtillieres

ACE, 16 (4.6) CC BY-ND 3.0 ES | UPC Barcelona, España | Reflexiones en torno a la forma urbana en la experiencia francesa de renovación urbana de los polígonos de vivienda masiva. DOI: http://dx.doi.org/10.5821/ace.16.46.9876 
Newman, O. (1972). Defensible Space: Crime Prevention through Urban Design. Macmillan.

Nika, J., y Cattin, A.L. (Eds.). (2017). Chevilly-Larue. ZAC Sorbiers-Saussaie. Groupe Valophis. En Repères. Aménagement, urbanisme. Densification des emprises foncières existantes: un nouveau gisement pour la production? Analyse de stratégies et pratiques d'organismes Hlm (Vol. 32, pp. 6169). Caisse des Dépôts - L’Union Sociale pour l'Habitat.

Panerai, P., y Mangin, D. (2002). Proyectar la ciudad. Celeste.

Roux, M.-C., Goeneutte, B., Malservisi, F., Kauffmann, V., Kossi, L., Delouvrier, F., y Jakubowski, T. (2019). Ensembles, l'histoire continue. Mémoire et projets des grands ensembles. En Cahier de la Maison de Banlieue et de l'Architecture (Vol. 26). Maison de Banlieue et de l'Architecture.

Rowlands, R., Musterd, S., y van Kempen, R. (Eds.). (2009). Mass Housing in Europe: Multiple Faces of Development, Change and Response. Palgrave Macmillan UK. DOI: https://doi.org/10.1057/9780230274723

Sendra, P. (2016). Infrastructures for disorder. Applying Sennett's notion of disorder to the public space of social housing neighbourhoods. Journal of Urban Design, 21 (3), 335-352. DOI: https://doi.org/10.1080/13574809.2015.1133223

Texier, S., y Radouan, S. (2010). Denis Honegger. Infolio, Éd. du Patrimoine.

Turkington, R., van Kempen, R., y Wassenberg, F. (Eds.). (2004). High-rise housing in Europe: Current trends and future prospects. Delft University Press.

Van Es, E., Harbusch, G., Maurer, B., Pérez, M., Somer, K., y Weiss, D. (Eds.). (2015). Atlas of the functional city. CIAM 4 and comparative urban analysis. THOTH Publishers/gta Verlag.

Wassenberg, F. (2012). Housing Estates. En S. J. Smith, M. Elsinga, L. F. O’Mahony, O. S. Eng, S. Wachter, y M. Pareja Eastaway (Eds.), International Encyclopedia of Housing and Home (Vol. 3, pp. 444-449). Elsevier. DOI: https://doi.org/10.1016/B978-0-08-047163-1.00530-0

Wassenberg, F. (2013). Large housing estates: Ideas, rise, fall and recovery. The Bijlmermeer and beyond. IOS Press - Delft University Press. 